\section{Therapeutic drug monitoring in epilepsy clinic: a multi-disciplinary approach}

\author{
Sunee Lertsinudom, 1 \\ Aporanee Chaiyakum, 1 \\ Supinya Tuntapakul,1 \\ Kittisak Sawanyawisuth,2,3 \\ Siriporn Tiamkao,4 Somsak Tiamkao,2,5 \\ Integrated Epilepsy Research Group 5 \\ 1Department of Clinical Pharmacy, \\ Faculty of Pharmaceutical Science, \\ 2Department of Medicine, Faculty of \\ Medicine, ${ }^{3}$ Department of Pharmacology, \\ Faculty of Medicine, 4The Research and \\ Training Center for Enhancing Quality of \\ Life of Working-Age People, 5Integrated \\ Epilepsy Research Group, Khon Kaen \\ University, Thailand
}

\section{Abstract}

Epilepsy is a common public health problem and needs multi-disciplinary treatment. Therapeutic drug monitoring (TDM) is one of step of the multi-disciplinary treatment in epilepsy at Epilepsy clinic, Khon Kaen University (Thailand). The TDM service has been established since 2008. Here, we aimed to study the roles of TDM order and epilepsy control. This is a prospective descriptive study in which data collection was done from January 1 to December 31, 2010, the period when pharmacists took part in assessing the appropriateness in measurement and interpretation of TDM in order to provide suggestions for physicians. The 112 patients under study had an average age of $38.21 \pm 15.36$ years; 254 samples were collected for therapeutic drug monitoring; phenytoin was submitted mostly for drug monitoring at $46.46 \%$; $44.49 \%$ of submissions for drug level monitoring were made owing to a suspected sub-therapeutic level. Associations were found between reasons of sending samples for drug level monitoring and the measured drug levels, i.e., $66.67 \%$ of drug levels found was so low that they were undetectable in sample for patients' compliance investigation and $38.94 \%$ of the drug levels were found to be sub-therapeutic as for the case where submission of samples was done because of suspected sub-therapeutic level, $40 \%$ of the cases were found to be in toxicity range in the cases with suspected over-therapeutic levels and monitoring levels, 58.25\% were found to be within the therapeutic range. Pharmacists used the interpreted results in patients' care by recommending physicians to monitor therapeutic drug closely, to adjust the dosage of drugs, and to recommend checking patients' compliance in their use of drugs at $56.5,38.9$, and $4.3 \%$, respectively. Physicians' responses were found to be absolute follow, partial follow and not follow at 77.95, 11.03, and $7.48 \%$, respectively. In conclusion, associations were found between reasons of TDM order and measured drug level. Therapeutic drug monitoring services at the Epilepsy Clinic was useful in supporting clinical information queries. Pharmacists could make use of interpreted drug level information by recommending physicians to monitor drug levels and adjust individual dosage regimen accordingly. It should be noted that physicians accepted pharmacists' recommendation, denoting multi-disciplinary care team that would lead to greater efficiency.

\section{Introduction}

Epilepsy is an important public health problem worldwide. Approximately 45 million people worldwide are diagnosed with epilepsy. In the United States, the prevalence of epilepsy is 6-8 cases/1000 people with the incidence of 26 40 cases/100,000 people annually. The common age ranges are children and adults age over 60 years. ${ }^{1}$ Antiepileptic drugs (AEDs) are the cornerstone treatment to control seizures resulting in occurrence of drug-related problems. In Thailand, $39.13 \%$ of persons with epilepsy (PWE) had drug interaction between phenytoin and other medications, while $28.99 \%$ of PWE had adverse drug reactions from AEDs. In addition, 10.14\% of PWE did not receive AEDs. ${ }^{2}$ The adverse events of AEDs may occur due to narrow therapeutic range such as phenytoin, carbamazepine, or valproic acid. Some AEDs also are metabolized via enzymatic system such as enzyme inducer (phenytoin, phenobarbital, and carbamazepine) or enzyme inhibitor (sodium valproic acid). Due to the AEDs properties, physicians need to be more aware on drug interaction, side effects, therapeutic level, and importantly seizure control. ${ }^{3}$

Pharmacists may play important role to drug related issues for PWE. ${ }^{4}$ Srinagarind Hospital is a university hospital, Khon Kaen University and located in the central of Northeastern, Thailand. The hospital is a tertiary care hospital and received referral uncontrolled cases of epilepsy. Pharmacists became involved in the Epilepsy Clinic, Srinagarind Hospital in 2005 resulting in lower cases of drug-related issues. In July 2009, the therapeutic drug monitoring system was established. The aim of this system is to provide multi-disciplinary treatment for PWE in regard of AEDs level monitoring, pharmacokinetic of AEDs, and drug interaction monitoring. Here, we reported the function of the therapeutic drug monitoring by pharma-
Correspondence: Somsak Tiamkao, Department of Medicine, Faculty of Medicine, Khon Kaen University, Khon Kaen, 40002, Thailand.

Tel: +66.433 .63664 - Fax: +66.433 .48399 .

E-mail: somtia@kku.ac.th

Key words: epilepsy, antiepileptic drugs, therapeutic drug monitoring.

Acknowledgements: this study was supported by TRF grants from Senior Research Scholar Grant, Thailand Research Fund grant number RTA5580004 and the Higher Education Research Promotion and National Research University Project of Thailand, Office of the Higher Education Commission, Thailand through the Health Cluster (SHeP-GMS), Khon Kaen University.

Contributions: the authors contributed equally.

Conflict of interests: the authors declare no potential conflict of interests.

Received for publication: 31 August 2014.

Revision received: 23 September 2014.

Accepted for publication: 30 October 2014 .

This work is licensed under a Creative Commons Attribution NonCommercial 3.0 License (CC BYNC 3.0).

(C) Copyright S. Lertsinudomet al., 2014

Licensee PAGEPress, Italy

Neurology International 2014; 6:5620

doi:10.4081/ni.2014.5620

cists at epilepsy clinic as a part of multi-disciplinary approach.

\section{Materials and Methods}

This study was conducted prospectively from January 1 to December 31, 2010 at Epilepsy Clinic, Khon Kaen University, Thailand. We studied all consecutive PWEs who participated the therapeutic drug monitoring. Data were collected in terms of the reasons for the therapeutic drug monitoring, interpretation of therapeutic drug level, pharmacists' recommendations, and physician practice.

The therapeutic drug monitoring system functioned as follows. When PWEs were uncontrolled or had drug-related events and required drug level monitoring, the patients were participated in the therapeutic drug monitoring system. The system comprised of attending physicians, pharmacists, and nurses. The team discussed on drug-related issues and seizure control. Pharmacists played roles in drug level interpretation and made clinical suggestion. Physician made final treatment after the group discussion. 
Data were analyzed by descriptive statistics in terms of reasons for therapeutic monitoring, association between the reason of TDM order and measured drug level, therapeutic drug monitoring interpretation, pharmacists' recommendations, and physician practice. The correlation between pharmacist recommendations and physician practices were classified into three categories; physicians completely follow the pharmacist recommendations, physicians partially follow the pharmacist recommendations, and physicians did not follow the pharmacist recommendations. The study protocol was approved by the ethics committee in human research, Khon Kaen University (HE460830).

\section{Results}

There were 112 PWEs participated the therapeutic drug monitoring system. The mean age of all patients was 38.21 years (SD 15.36) and 51 patients (45.54\%) were male. The drug level monitoring was performed 254 times. Most patients (83 patients, $74.10 \%$ ) had one or two times of drug level monitoring (range 1-12 times) as shown in Table 1. Therapeutic drug monitoring was commonly performed in PWEs with phenytoin and due to sub-therapeutic reason in $46.46 \%$ and $44.49 \%$ (Tables 2 and 3 ).

None of PWEs who performed therapeutic drug monitoring due to compliance check had drug level in therapeutic level, while only 53 patients (46.90\%) in sub-therapeutic suspicious group had therapeutic level of AEDs. Fourteen patients $(40.00 \%)$ who were suspected to have toxicity had AEDs in toxic level. Only 60 patients $(58.25 \%)$ of patients who performed drug level as a routine manner had therapeutic level of AEDs (Table 4). Of the 254 AEDs monitoring, 245 samples (96.46\%) were interpreted and suggested recommendation by pharmacists. There were 329 recommendations for the 245 samples (each individual result may have more than one recommendation). The most common recommendation was follow-up drug level in 186 samples (75.92\%) due to dosage adjustment, inconsistent monitoring values with clinical, non-therapeutic drug levels, and uncontrolled seizures (Table
5). The other 9 samples were not interpreted due to AEDs changing (1), wrong AEDs (1), and unknown reason (7). Physicians completely followed the pharmacists' recommendation in most monitoring (198 times, 80.82\%) as shown in Table 6 .

\section{Discussion and Conclusions}

Seizure control with no side effect from AEDs is the main goal for epilepsy treatment. Therapeutic drug monitoring with multi-disciplinary approach is a strategy that may improve epilepsy treatment. Holistic patient discussion in various aspects particularly drug interactions and side effects may lessen AEDs side effect.

Phenytoin is the most common AEDs in Thailand due to its low cost and good efficacy. It may however have drug interaction and side effects such as hepatitis. PWEs who participated the therapeutic drug monitoring were mostly taking phenytoin (46.46\%) and sodium valproate $(29.53 \%)$. These may imply that phenytoin may have issue with seizure control or AEDs related side effects. A previous study from Thailand also showed that $55.8 \%$ of PWEs that required drug monitoring were taking phenytoin..$^{5}$ In the present study, only half of patients (50.79\%) had AEDs level in the therapeutic range (Table 4). This information is compatible with other studies. Affolter et al. and Nakariyakul found that $49.3 \%$ and $55.0 \%$ of PWEs had AEDs level in the therapeutic range.6,7 The association of AEDs levels and clinical presentation of uncontrolled PWEs or PWEs with drug issues were shown in Table 4. The correlations of AEDs levels with clinical problems are approximately 40\%. Sub-therapeutic AEDs levels were shown in $38.94 \%$ of PWEs with clinical issue of sub-therapeutic suspicion, while $40 \%$ of clinical suspicious of toxicity had AEDs levels at toxic level. Clinicians therefore should not be confident with the clinical presentation. We would recommend to have AEDs level monitoring in PWEs with uncontrolled seizures because using clinical features may correct in only $40 \%$ of patients. These rules may apply only if poor compliance is suspected because all three patients who had poor compliance showed low level of AEDs. When work as a multi-disciplinary team, almost $100 \%$ of AEDs levels were interpreted by pharmacists $(96.46 \%)$ with recommendations. Physicians decided treatment for individual PWEs based on the recommendations and discussion with the team. Due to multidisciplinary approach, team members discussed the results, recommendations, and clinical of the patient. The decision was made based on the clinical status of the patients. There were $80.82 \%$ of team decision followed the pharmacists' recommendations which was

Table 1. Numbers of time of antiepileptic drugs (AEDs) level monitoring.

\begin{tabular}{lc}
$\begin{array}{l}\text { lime of AEDs level } \\
\text { monitoring }\end{array}$ & Patients (\%) \\
Once & $43(38.39)$ \\
Twice & $40(35.71)$ \\
\hline 3 times & $10(8.93)$ \\
4 times & $11(9.82)$ \\
\hline 5 times & $1(0.89)$ \\
6 times & $4(3.57)$ \\
\hline 8 times & $2(1.79)$ \\
12 times & $1(0.89)$ \\
\hline
\end{tabular}

Table 2. Submission of blood samples for therapeutic drug monitoring classified by antiepileptic drug $(n=254)$.

\begin{tabular}{lc} 
Antiepileptic drugs & Samples (\%) \\
Carbamazepine & $29(11.42)$ \\
Phenobarbital & $32(12.59)$ \\
\hline Phenytoin & $118(46.46)$ \\
Sodium valproate & $75(29.53)$
\end{tabular}

Table 3. Reasons for therapeutic drug monitoring $(n=254)$.

\begin{tabular}{lc} 
Reasons for therapeutic & Samples (\%) \\
drug monitoring & \\
Checking compliance & $3(1.18)$ \\
Suspected sub-therapeutic & $113(44.49)$ \\
\hline Suspected toxicity & $35(13.78)$ \\
Routine monitoring & $103(40.55)$ \\
\hline
\end{tabular}

Table 4. Association between reasons for therapeutic drug monitoring order and measured drug levels.

\begin{tabular}{|c|c|c|c|c|c|c|}
\hline \multirow[t]{2}{*}{ Reasons for TDM order } & \multicolumn{6}{|c|}{ Measured level: number of samples (\%) } \\
\hline & Therapeutic level & Sub-therapeutic level & Toxic level & Undetectable & Unknown & Total \\
\hline Checking compliance & - & $1(33.33)$ & - & $2(66.67)$ & - & 3 \\
\hline Suspected sub-therapeutic & $53(46.90)$ & $44(38.94)$ & $14(12.39)$ & $2(1.77)$ & - & 113 \\
\hline Suspected toxicity & $16(45.71)$ & $5(14.29)$ & $14(40.0)$ & - & - & 35 \\
\hline Routine monitoring & $60(58.25)$ & $26(25.24)$ & $11(10.68)$ & $5(4.85)$ & $1(0.97)$ & 103 \\
\hline Total & $129(50.79)$ & $76(29.92)$ & $39(15.35)$ & $9(3.54)$ & $1(0.39)$ & 254 \\
\hline
\end{tabular}


Table 5. Pharmacists' recommendations $(n=239)$.

\begin{tabular}{lc} 
Pharmacists' recommendations & N. of recommendation (\%) \\
Adjustment of dosage regimen & $128(38.91)$ \\
Changing medication & $1(0.30)$ \\
\hline Monitoring drug level at next visit & $186(56.53)$ \\
Checking patient compliance & $14(4.26)$ \\
\hline
\end{tabular}

Recommendations for each individual may have more than one recommendation; the total number of sample for antiepileptic monitoring was 245 .

Table 6. Physicians' response to pharmacists' recommendations (n=239).

\begin{tabular}{lc} 
Physicians' response & Samples (\%) \\
Completely follow to recommendation & $198(80.82)$ \\
Partially follow to recommendation & $28(11.43)$ \\
\hline Not follow to recommendation & $19(7.76)$ \\
\hline
\end{tabular}

somewhat lower than the previous report by Nakariyakul (2009). ${ }^{6}$ This multi-disciplinary team may be suitable for the tertiary care hospital where PWEs are always difficult and complicated.

In conclusion, therapeutic drug monitoring at the Epilepsy Clinic by a multi-disciplinary approach is an important strategy to identify causes of uncontrolled epilepsy control more correctly.

\section{References}

1. French JA, Pedley LA. Initial management of epilepsy. N Engl J Med 2008;359:166-76.

2. Kanjanasilp J, Preechagoon Y, Kaewvichit S, Edward Richards RM. Pharmaceutical care improved outcomes in epileptic patients. CMU J Nat Sci 2008;7:33-45.

3. Limphan J, Lertsinudon S. Antiepileptic hypersensitivity syndrome: implication on pharmaceutical care. In: Sonthisombat $\mathrm{P}$, ed. Contemporary review in pharmacotherapy 2011. Phitsanulok: The College of Pharmacotherapy of Thailand; 2011. pp 75-97.

4. Lertsinudon S, Chaiyakham A, Tantapakul S, Tiamkao S. Pharmacist's intervention on managing drug related problems in epilepsy clinic, Srinagarind Hospital. North-Eastern Thai J Neurosci 2009;4:3950.

5. Ratanajamit C, Kaewpibal P, Satthawacharavanich S, Faroongsamg D. Effect of pharmacist participation in the health care team on therapeutic drug monitoring: Utilization for antiepileptic drugs. J Med Assoc Thai 2009;92:1500-7.

6. Nakariyakul K. Reasons and acceptance of physicians in their sending of blood samples for therapeutic drug level monitoring in pharmacological outpatient treatment. Hosp Pharm J 2009;19:S18-25.

7. Affoiter N, Krahenbuhl S, Schienger RG. Appropriateness of serum level determinations of antiepileptic drugs. Swiss Med Wkly 2003;133:591-7. 